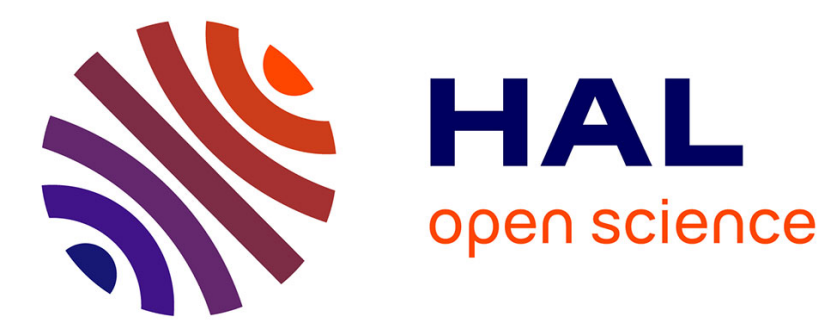

\title{
Vers un management des tensions de gouvernance publique dans les établissements de santé ?
}

\author{
Laurent Mériade, Corinne Rochette, Damien Talbot
}

\section{To cite this version:}

Laurent Mériade, Corinne Rochette, Damien Talbot. Vers un management des tensions de gouvernance publique dans les établissements de santé ?: UNE PROPOSITION DE REPONSE PAR LA PROXIMITE. Innovations - Revue d'économie et de management de l'innovation, 2019, $\mathrm{N}^{\circ} 60$ (3), pp.169. 10.3917/inno.060.0169 . hal-02289309

\section{HAL Id: hal-02289309 \\ https://hal.science/hal-02289309}

Submitted on 4 Dec 2019

HAL is a multi-disciplinary open access archive for the deposit and dissemination of scientific research documents, whether they are published or not. The documents may come from teaching and research institutions in France or abroad, or from public or private research centers.
L'archive ouverte pluridisciplinaire HAL, est destinée au dépôt et à la diffusion de documents scientifiques de niveau recherche, publiés ou non, émanant des établissements d'enseignement et de recherche français ou étrangers, des laboratoires publics ou privés. 


\title{
VERS UN MANAGEMENT DES TENSIONS DE GOUVERNANCE DE PUBLIQUE DANS LES ETABLISSEMENTS DE SANTE ? UNE PROPOSITION DE REPONSE PAR LA PROXIMITE
}

\author{
Laurent MERIADE, Corinne ROCHETTE, Damien TALBOT \\ IAE d'Auvergne- Université Clermont Auvergne-Chaire «Santé et Territoires » \\ laurent.meriade@uca.fr
}

\begin{abstract}
Résumé
Les tensions de gouvernance publique, définies comme des contradictions, oppositions voire des complémentarités entre les logiques de management et les pratiques publiques (Bartoli et al., 2011) sont, depuis une vingtaine d'années, particulièrement apparentes dans les établissements de santé français. Mazouz et Tardif (2010) ont proposé un cadre explicatif de ces tensions à quatre niveaux d'analyse qui portent sur les institutions, l'organisation, le cadre de gestion et les outils de gestion. En revanche, ce modèle fournit peu d'explications sur la manière dont, dans les établissements, ces tensions apparaissent et pourraient être gérées. Aussi, l'objet de cet article est de combler ce manque en cherchant à identifier les facteurs d'apparition puis éventuellement de gestion des tensions de gouvernance publique dans les établissements de santé en France. Pour cela, nous mobilisons les travaux de l'Ecole de la Proximité qui reposent sur l'affirmation que plus les acteurs sont proches (et ce de diverses manières), plus la probabilité qu'ils interagissent est forte (Torre et Rallet, 2005 ; Boschma, 2005 ; Talbot, 2008) et qu'ils parviennent à gérer ou atténuer les tensions. Nous appliquons conjointement ces deux cadres d'analyse au cas d'un Centre de Lutte Contre le Cancer (CLCC) français. Nos premiers résultats mettent en évidence des pistes de compréhension de l'apparition de ces tensions à partir de proximités propres aux établissements de santé.
\end{abstract}

Mots clés : Etablissements de santé, tensions, proximités, Centre de lutte contre le cancer, management

\begin{abstract}
Public governance tensions, defined as contradictions, oppositions or even complementarities between management logics and public practices (Bartoli et al. 2011), have been particularly apparent in French healthcare institutions for the past twenty years. Mazouz and Tardif (2010) proposed a framework to explain these tensions at four levels of analysis that focus on institutions, organization, management framework, and management tools. However, this model provides little explanation of how these tensions could be managed in institutions. The purpose of this article is to fill this gap by seeking to identify the possibilities of managing public governance tensions in health care institutions in France. To do this, we mobilize the work of the School of Proximity that the closer, in various ways, the closer the actors are, the more likely they are to interact (Torre and Rallet, 2005 ; Boschma, 2005 ; Talbot, 2008). We apply these two analytical frameworks jointly to the case of a French Cancer Control Centre (CLCC). Our initial results highlight ways to better understand and manage these tensions based on the proximity of healthcare facilities.
\end{abstract}

Keywords: Health establishments, tensions, proximity, CLCC, management 


\section{VERS UN MANAGEMENT DES TENSIONS DE GOUVERNANCE DE PUBLIQUE DANS LES ETABLISSEMENTS DE SANTE? UNE PROPOSITION DE REPONSE PAR LA PROXIMITE}

En France, dans les établissements de santé, les ordonnances Juppé (1996), puis l'instauration progressive de la Tarification à l'Activité (T2A - 2004) ont progressivement instiller une logique de résultats inspirée par les préceptes du New Public Management (Hood, 1991).

Aujourd'hui, après une vingtaine d'années de mise en œuvre progressive de la gestion par les résultats (Mazouz, 2012) dans ces organisations, il est possible d'identifier diverses formes de tensions de gouvernance publique (Bartoli et $a l, 2012 \mathrm{a}$ ) qui impactent de manière significative l'exercice quotidien des activités des personnels. Ces tensions se manifestent soit par diverses formes de blocages et de contournements (Walker et al, 2010, Carassus et al., 2012), soit, de manière moins apparente, par des insatisfactions internes comme externes (Rivière et al, 2013). Dans le secteur de la santé, leurs causes ont été déjà illustrées de manière très exhaustive (Grenier et Ibrahim, 2015). La variété et la superposition de logiques différentes (institutionnelles et professionnelles) (Pierru, 2012), la diversité des parties prenantes et la dispersion des nœuds de pouvoir (Grenier, 2009), les pressions financières (Bérard et al. 2015), les conflits éthiques (Bérard, 2010), les conflits entre innovation et normalisation des pratiques hospitalières et médicales (Marival et al. 2015) sont les principales tensions recensées dans la littérature récente. En revanche, dans le contexte macro et micro économique actuel des établissements de santé, aux yeux des gestionnaires comme des personnels, ces déterminants apparaissent souvent difficilement surmontables ou gérables.

Le particularisme inhérent à ces tensions mais aussi la difficulté à traiter ce sujet résident dans leur grande diversité. Pour faciliter leur analyse, Mazouz et Tardif (2010) identifient des tensions de gouvernance publique (TGP) et recensent quatre niveaux d'étude de ces tensions : les institutions, l'organisation, le cadre de gestion et les outils de gestion.

Cette approche constitue une heuristique d'analyse intéressante qui permet une catégorisation, certes simplificatrice, mais suffisamment large et opérationnelle pour améliorer notre lecture de ces tensions. En revanche, elle fournit peu d'explications sur la manière dont, dans les établissements, ces tensions pourraient être atténuées ou gérées notamment de manière prophylactique. Aussi, l'objet de cet article est de combler ce manque en cherchant à identifier les facteurs d'apparition, puis éventuellement de gestion, des tensions de gouvernance publique qui se manifestent de manière atténuée ou amplifiée dans les établissements de santé en France.

Ce questionnement est d'autant plus intéressant qu'il permet de s'interroger, par des approches non encore explorées, sur les freins ou les résistances à l'innovation dans ces organisations.

Pour cela, nous proposons de mobiliser les travaux de l'École de la Proximité. Cette Ecole étudie les conditions de l'apparition, du maintien voire de la destruction des relations économiques (Bellet et al., 1993 ; Torre et Gilly, 2000 ; Pecqueur et Zimmermann, 2004 ; Boschma, 2005 ; Torre et Rallet, 2005 ; Knoben et Oerlemans, 2006 ; Rychen et Zimmermann, 2008 ; Carrincazeaux, Grossetti et Talbot, 2008 ; Balland et al., 2014). Elle montre que plus les acteurs sont proches, plus la probabilité qu'ils nouent une relation est forte (Cassi et Plunket, 2014). Elle identifie également des formes de proximités non spatiales (Bellet et al., 1993 ; Torre et Gilly, 2000) qui peuvent prendre des formes institutionnelles, organisationnelles, managériales ou encore sociales (Boschma, 2005) et qui constituent le socle du développement de relations organisationnelles. Ces formes de proximités décrivent les manières dont les acteurs nouent des relations entre eux (Mériade et al. 2017). Leurs absences mais aussi parfois, paradoxalement, leurs trop grandes intensités peuvent expliquer l'apparition de tensions dans les établissements de santé (ibid.) dans la mesure où elle permet 
d'explorer plus finement les tensions et leurs origines. Les proximités constituent une grille d'analyse qui mérite d'être explorée. Nous proposons donc d'associer ce cadre d'analyse à celui des TGP (Mazouz et Tardif, 2005) en les appliquant au cas d'un Centre de Lutte Contre le Cancer (CLCC) français.

Pour cela, notre travail s'organise de la façon suivante : dans une première et une deuxième partie, nous revenons successivement sur les définitions des tensions dans les organisations de santé et sur les proximités, puis nous présentons nos deux cadres théoriques et leurs complémentarités. Nous précisons la méthodologie retenue et la nature de nos données dans une troisième partie, puis nous exposerons et discuterons nos résultats dans une dernière partie avant de conclure.

\section{Les tensions de gouvernance publique (TGP)}

La gouvernance des établissements hospitaliers se caractérise par une relation au moins tripartite (administrateurs, médecins, gestionnaires) qui impacte l'ensemble du fonctionnement et des relations au sein de ces organisations. Dans le milieu de la santé, elle fait naitre de nombreuses tensions de gouvernance publique (Bartoli et al., 2012a) et déviances organisationnelles (Carassus et al., 2012) qu'une analyse descriptive des relations interpersonnelles peut permettre d'identifier voire de gérer à travers ses principaux déterminants. Aujourd'hui, les praticiens hospitaliers sont fortement mobilisés par des tâches administratives pour lesquelles ils communiquent et négocient, plus que hier, avec les administrateurs et gestionnaires de leurs établissements. Si des tensions de rôle sont identifiées et subies par ces acteurs face auxquelles ils sont tenus de s'adapter (Pierru, op.cit.), un processus de défiance/attirance semble progressivement les caractériser, ce qui n'est pas sans incidence sur leur manière d'appréhender leurs relations avec les autres catégories de personnels (Georgescu et Naro, 2012).

Les tensions de gouvernance sont identifiées dans la littérature comme le résultat de nombreuses injonctions paradoxales (Bartoli et al., 2012b). En milieu de santé, elles sont particulièrement importantes. La confrontation des impératifs sociaux et sociétaux de l'action publique avec son utilitarisme ou sa rentabilité, la spécificité même des missions de services publics et la rationalisation des moyens pour les mener à bien, l'objectif de professionnalisation des personnels et l'obligation de reconnaissance des établissements, l'impératif d'autonomie des organisations et la volonté de maintenir une égalité d'accès et de traitement des patients sur tout le territoire constituent quelques exemples d'injonctions paradoxales qui accompagnent la mise en œuvre de la gestion par résultats dans les organisations de santé et conduisent au développement de tensions autour des formes de gouvernance publique. L'identification de ces tensions constitue une approche riche de perspectives car elle doit nous permettre de circonscrire leurs principales manifestations mais, aussi et surtout, leurs déterminants. Ces tensions s'expriment de manière magistrale dans les organisations de santé dans lesquelles naissent des oppositions entre les impératifs des missions de service public et ceux de leur utilitarisme et rentabilité. Ces tensions interrogent également la légitimité de la performance publique (Weick, 2009) et ouvrent le débat de la coexistence des valeurs de nature normative/quantitative dans les organisations. L'apparition de tensions de gouvernance publique va en effet conduire les personnels de santé à adapter, gérer et parfois faire évoluer à la fois leurs représentations et leurs pratiques (Mazouz et Tardif., 2010). C'est par l'étude de ces perceptions et pratiques que ces auteurs ont développé leur cadre d'analyse des TGP et mis en évidence quatre formes de tensions : éthique, structurelle, managériale et instrumentale.

Les tensions institutionnelles se manifestent par des préoccupations d'ordre éthique et relèvent d'une opposition entre les valeurs, le sens attribués à l'action publique et les modes 
opératoires associés à la gestion de la performance (Bartoli et al., 2011). Elles renvoient au sens et à la cohérence qui fondent la légitimité de la performance dans le contexte public. Elles amènent à réinterroger la place de l'éthique dans un contexte d'évolution des valeurs et des croyances collectives portées par les institutions publiques (Mazouz et al., 2012; Mériade, 2017). Elles renvoient à la difficulté pour les agents publics de faire cohabiter les valeurs publiques et les objectifs de performance.

Parallèlement ou de manière diachronique, des tensions organisationnelles se manifestent de manière plus conjoncturelle. Elles affectent surtout le partage du pouvoir et les formes de coordination de l'action publique. Les tensions managériales apparaissent en réponse à des changements initiés au niveau des procédures et des cadres de gestion de l'action publique. La gestion par les résultats, que les réformes récentes ont activée par substitution à la gestion des moyens (Mazouz et al., op.cit. ; Mériade, op.cit.), représente un de ces changements dont les incidences s'exercent structurellement sur la manière dont les innovations managériales vont être utilisées par les acteurs.

Enfin, les tensions artefactuelles concentrent les conséquences instrumentales des tensions précédentes. Elles sont décrites comme des incompatibilités évidentes entre les outils de gestion effectivement utilisés et le cadre de gestion prescrit (Mazouz et al, op.cit. ; Mériade, op.cit.). Elles naissent des zones de contacts entre les nouveaux outils de gestion et les valeurs, les structures ou le management et se manifestent par des adaptations, des rejets ou encore des détournements instrumentaux.

Ces quatre formes de tensions permettent de bien décrire l'ensemble des tensions apparaissant dans une organisation. A l'hôpital, outre le fait qu'elles trouvent leurs origines dans l'évolution du cadre de l'action publique, elles s'ancrent aussi dans la nature même des mondes qui cohabitent dans ces organisations particulièrement complexes que sont les hôpitaux. Glouberman et Mintzberg $(2001$; 2002) décrivent précisément les quatre mondes que sont le traitement (cure) dont la figure est le médecin, les soins (care) représentés par les infirmières, le contrôle incarné par les gestionnaires et la communauté que personnifient les administrateurs. Les auteurs proposent une lecture de la dynamique des relations entre ces quatre mondes en s'appuyant sur la nature des tâches et l'engagement envers le patient: le traitement ponctuel (épisode aigu) et programmé pour les médecins, la coordination complexe et continue pour le personnel infirmier, la mesure et le contrôle des ressources, des budgets pour les gestionnaires qui constituent une hiérarchie de bureau bien moins influente que celle médicale et enfin la surveillance (toute relative) des activités par les administrateurs qui exercent une pression sur les gestionnaires. Ces mondes renvoient à "des valeurs non reconciliées »(Glouberman et Mintzberg, 2002) et des «structures incompatibles » malgré les coalitions qui s'établissent (coalition clinique entre médecins et infirmières, coalition de statut entre médecins et administrateurs, coalition de limitation entre administrateurs et gestionnaires et coalition d'initiés entre gestionnaires et infirmières). Ces coalitions se nouent à travers des formes de proximités même si les auteurs ne les expriment pas toujours dans ces termes.

Ainsi, il apparait que l'analyse des proximités peut renforcer et compléter le cadre d'analyse des tensions de gouvernance publique qui, pour l'instant, ne fournit pas une force compréhensive suffisante pour permettre de promouvoir une identification et gestion proactive de ces tensions dans les établissements de santé.

\section{L'école de la proximité}

L'approche de l'Ecole de la Proximité a notamment été utilisée pour traiter des relations interindividuelles en général (Grossetti, 2008) et des modalités de construction de la confiance interpersonnelle dans les organisations en particulier (Dupuy et Torre, 2004). Plus précisément, les travaux portant sur la spatialité des organisations (Knoben et Oerlemans, 
2006) suggèrent que pris isolément, l'espace géographique (l'« espace vide » selon Lefebvre, 2007) ne peut garantir des échanges de connaissances et d'informations. L'espace doit être aussi entendu comme le produit de pratiques sociales (Torre et Rallet, 2005). La littérature restitue les caractéristiques sociales de l'espace dans la diversité des formes de proximités non spatiales (Bellet et al., 1993 ; Torre et Gilly, 2000), aujourd'hui identifiées dans les travaux en sciences de gestion (Ingham et al., 2011; Nilsson et al., 2015 ; Knoben et Oerlemans, 2006 ; Wilson et al., 2008).

Les travaux sur la proximité ne définissent pas la proximité par elle-même, mais à travers ses dimensions. L'approche par la proximité est une grille de lecture qui se nourrit de divers courants théoriques (institutionnalisme, interactionnisme, évolutionnisme, théorie de la régulation, théorie des organisations, etc.) pour poser la question de l'influence de la localisation des acteurs sur leurs interactions. Deux types de localisation sont traditionnellement envisagés : localisation dans l'espace géographique et localisation dans l'espace social. Les quatre mondes de l'hôpital constituent des espaces sociaux particulièrement marqués (Glouberman et Mintzberg, 2001) construits sur des formes de proximités.

Boschma (2005) propose de distinguer cinq formes de proximités qui circonscrivent de manière très robuste leurs diversités. Premièrement, la proximité géographique qui se définit simplement comme la distance métrique et/ou temporelle qui sépare les acteurs. On est proche géographiquement pour des raisons objectives ou subjectives. Deuxièmement, l'appartenance à une même organisation (firme, collectivité publique, laboratoire de recherche, université, hôpital, mais aussi réseau, chaîne de valeur, industrie, etc.) qualifie la proximité organisationnelle. Elle « [...] lie les agents participant à une activité finalisée dans le cadre d'une structure particulière. [...] [Elle] se déploie à l'intérieur des organisations - firmes, établissements, etc.- et, le cas échéant, entre organisations liées par un rapport de dépendance ou d'interdépendance économique ou financière - entre sociétés membres d'un groupe industriel ou financier, au sein d'un réseau, etc. » (Kirat et Lung, 1995, p. 213). Son existence permet de réduire l'incertitude inhérente à toute relation et de maîtriser l'opportunisme des individus. Troisièmement, la proximité cognitive renvoie au partage par les individus d'une base de connaissances similaires et/ou complémentaires. Elle ouvre la voie à une compréhension mutuelle. Son existence est à l'origine des relations d'apprentissage (Cassi et Plunket, 2014). Quatrièmement, parce que les relations économiques sont encastrées dans un réseau social (Granovetter, 1985), il est nécessaire de tenir compte de l'appartenance des individus à un même réseau. Les liens d'amitié et de parenté qui animent ce dernier fluidifient les interactions et réduisent les conflits et sont constitutifs d'une proximité sociale. Enfin, et cinquièmement, le partage d'institutions formelles et informelles comme des lois, des règles, des coutumes, des valeurs, etc. conditionnent les interactions en leur fournissant un cadre stable (Kirat et Lung, 1999) : on parle alors de proximité institutionnelle.

La mobilisation de ces cinq formes de proximité semble à la fois inédite et très appropriée à une analyse explicative et compréhensive de l'apparition des TGP dans les établissements de santé.

\section{Repères méthodologiques et éléments de présentation du cas}

Ce travail constitue la première phase d'un programme de recherche, déployé sur quatre ans, sur le management des organisations de santé développé avec un Centre de Lutte Contre le Cancer (CLCC par la suite). Le programme co-construit entre les acteurs de la santé et des chercheurs en gestion consiste, pour une partie importante, à observer les évolutions organisationnelles que connait l'hôpital et la manière dont les univers professionnels collaborent pour faire émerger des projets et de nouvelles formes d'organisation. C'est dans la 
perspective de la future émergence de ces coopérations que le groupe de recherche à suggérer de développer une première analyse des tensions existantes notamment en mobilisant le cadre de l'Ecole de la Proximité.

Les CLCC sont des établissements de santé privés à but non lucratif qui participent au service public hospitalier en France. Ils appartiennent au champ des Etablissements de Santé Privés d'Intérêts Collectifs (ESPIC). Créés en octobre 1945, ils sont financés par l'assurance maladie et sont contrôlés par le ministère de la Santé, dans les mêmes conditions que les hôpitaux publics. Ils assurent des missions de prévention, de recherche, d'enseignement et de soins. La particularité des CLCC réside dans leur modèle de prise en charge globale et multidisciplinaire des personnes atteintes d'un cancer. Ceci nécessite, plus qu'ailleurs encore, des interactions et des communications entre des acteurs présentant des profils professionnels très diversifiés. La direction de ces établissements est assurée par un directeur, médecin, assisté d'un secrétaire général et d'une équipe de direction qui fait appel à une diversité de cultures professionnelles (gestionnaire, médicale, soignante, etc.). La direction générale assurée par un médecin constitue une particularité forte des CLCC qui les distingue des CHU et dans la grande majorité des cas des cliniques privées.

Le Centre Jean PERRIN, fondé en 1973, est un des 18 CLCC, regroupés sous l'égide de la fédération nationale Unicancer. Reconnu comme pôle d'excellence dans sa spécialité, le CLCC Jean Perrin en quelques chiffres clés ${ }^{1}$, c'est : 712 salariés, 84,6 millions d'euros de recettes et 83,3 millions de dépenses soit un résultat de 1,2 millions d'euros, 26.965 consultations et 15.623 patients pris en charge par 60 spécialistes.

Il entretient depuis son ouverture une relation ambivalente avec le CHU avec lequel il partage : une même localisation géographique, la prise en charge régionale de la cancérologie (soins, recherche et enseignement) et les soins de suite des patients sur les centres hospitaliers de périphérie. De fait, si une coopération existe, en raison de leur proximité géographique, les deux établissements n'ont jamais développé de projet institutionnel ou médical commun. Pourtant, l'évolution du contexte hospitalier se traduit par la diffusion d'une logique de concurrence qui s'exprime dans les regroupements massifs de cliniques privées ou le rapprochement des établissements publics. Le plan Juppé (1996) de maitrise des dépenses de santé qui introduit un quasi marché, le système de financement dit T2A (tarification à l'activité), la loi HPST (2009), la création des GHT (groupements hospitaliers de territoire) en 2016 sont emblématiques de cette "évolution des pouvoirs parfois au détriment du corps médical » (Angelé-Halgand et Garrot, 2015, p. 50).

Le travail de recherche entrepris dans cet établissement réunit un collectif de chercheurs en gestion, les dirigeants (direction générale et des services) et des représentants du personnel (médecins, praticiens, logisticiens) qui constitue une véritable communauté épistémique. Il permet d'accéder de manière privilégiée aux matériaux (données quantitatives, réalisation d'entretiens libres et semi-directifs), de participer à l'ensemble des réunions relatives aux projets et à la stratégie de ce Centre à l'issue desquelles nous réalisons un compte rendu systématique. Les observations réalisées dans le cadre de notre participation à ces réunions ou échanges permettent de mieux appréhender le positionnement des groupes d'acteurs et des acteurs eux-mêmes dans la dynamique collective ou les signes de tensions et ainsi d'affiner les catégories de personnels sur lesquelles se concentrent les tensions. Ainsi nous avons retenu quatre catégories de personnels à interroger pour cette première étape de recherche : les dirigeants, les médecins, les personnels soignants et les personnels supports. Nos catégories se distinguent en partie des quatre mondes de Glouberman et Mintzberg (2001). En effet, il apparait clairement que les médecins endossent aussi l'habit de gestionnaire (Vallette et Burelier, 2014) de même que certains personnels soignants (par exemple les infirmières de

\footnotetext{
${ }^{1}$ Les chiffres sont extraits du rapport d'activité 2017.
} 
coordination), aussi nous n'avons pas retenu une catégorie gestionnaire mais une catégorie fonction support (service de gestion des ressources humaines, servie logistique, direction des services informatiques, etc.). La figure 1 reprend une carte des tensions issues de notre analyse de ces réunions.

Figure 1 : Carte des tensions entre les catégories de personnels issue de l'analyse des réunions (source : auteurs)

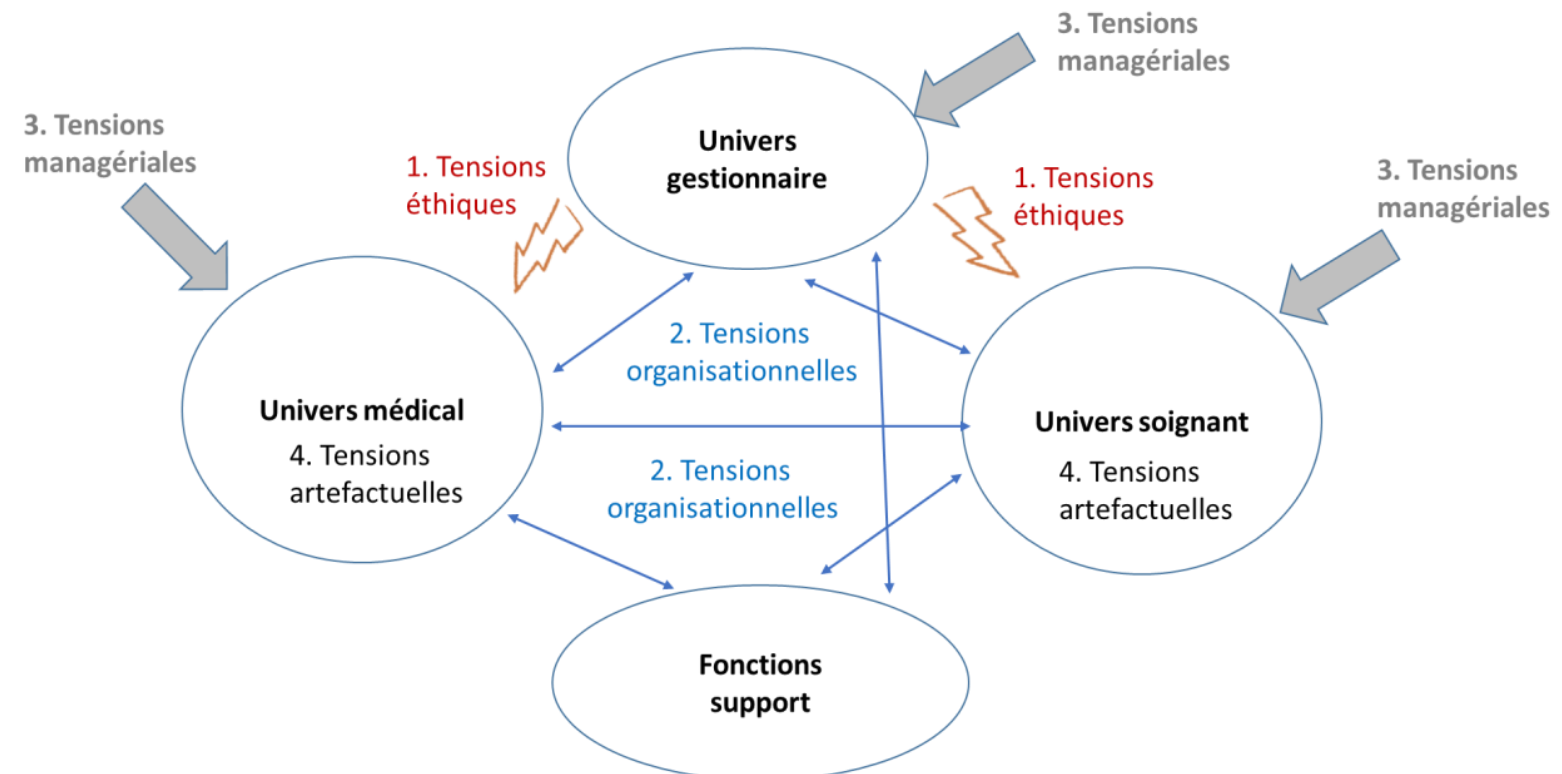

Notre objectif est ici de tester et évaluer le pouvoir explicatif de la grille de la proximité sur l'apparition des tensions de gouvernance dans cet établissement. Aussi, nous formulons la proposition générale que l'existence de proximités ou de distances entre les acteurs hospitaliers peut expliquer l'apparition ou la réduction de tensions dans les établissements. La figure 2 schématise la présence de proximités comme une explication à l'existence de tensions atténuées. Ainsi par exemple, un langage commun et des liens de parentés entre l'univers médical et gestionnaires ainsi qu'entre l'univers soignant et gestionnaire permettraient d'atténuer les tensions organisationnelles, managériales voire artefactuelles. L'existence d'un but partagé et clairement identifié ainsi que l'existence de règles et valeurs communes contribueraient à amenuiser les tensions organisationnelles et éthiques. Dans l'univers de l'hôpital, on peut supposer que la meilleure prise en charge possible du patient constitue un but sur lequel chacun se retrouve. Afin d'évaluer la pertinence de notre proposition générale nous avons utilisé ce cadre d'analyse comme grille de lecture des matériaux collectés sur le terrain.

Figure 2 : Proposition d'une articulation entre les tensions et les proximités (source : auteurs) 


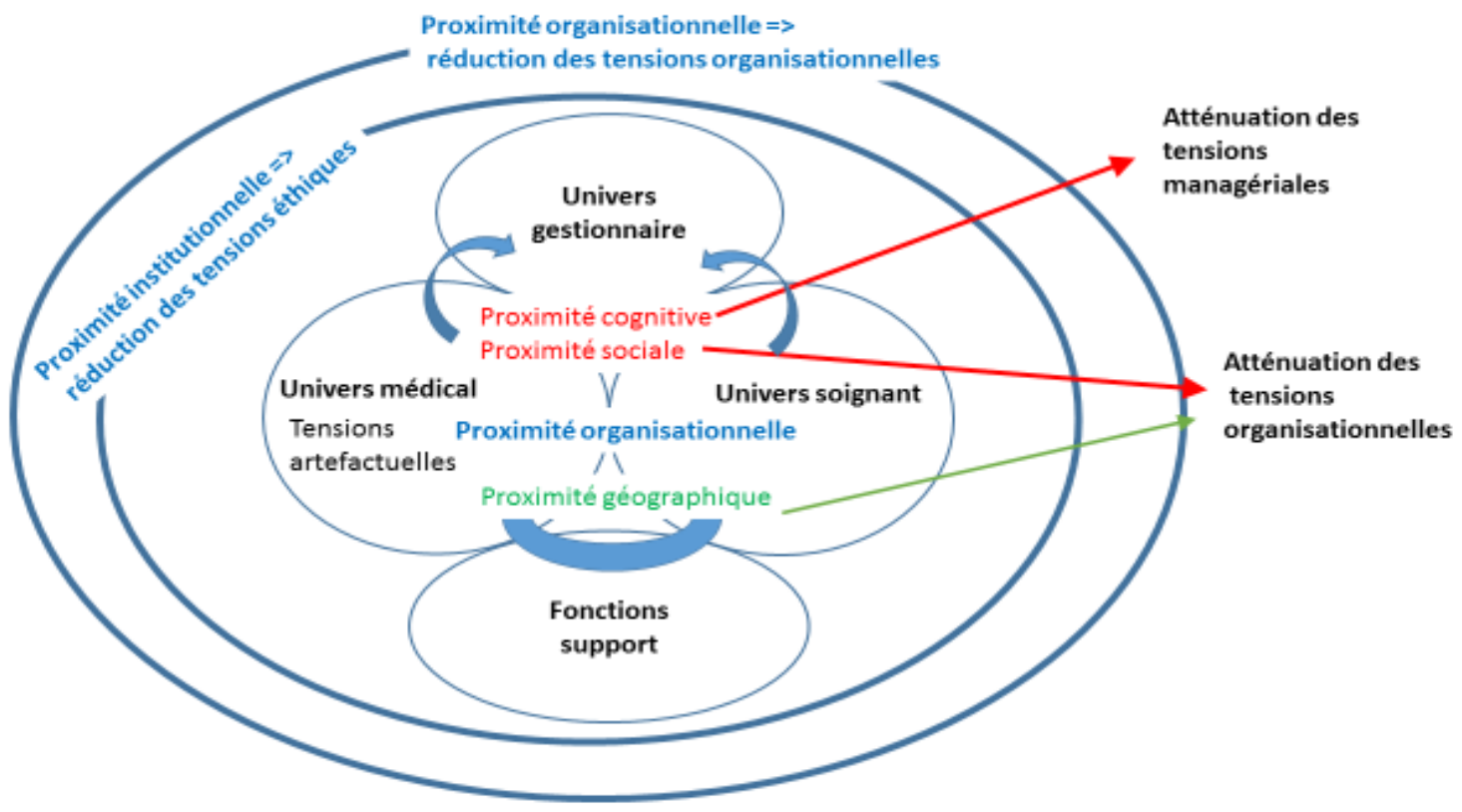

Nous nous appuyons plus précisément sur des données primaires ; les comptes rendu de cinq réunions et une série de huit entretiens semi directifs réalisés du mois de juin 2017 (durée moyenne de 44 minutes) conduits auprès des personnels du CLCC à partir d'un guide d'entretien identique (Annexe 1). Les entretiens enregistrés et retranscrits ont fait l'objet d'une analyse de contenu avec un codage des propos autour d'une grille reprenant les quatre catégories de tensions de Mazouz et al. (2012) et les cinq formes de proximité de Boschma (2005). Pour illustrer nos résultats nous nous appuierons plus précisément sur les propos de huit personnes interrogées (tableau 1). Nous avons réalisé au total trente entretiens auprès des personnels de l'établissement. Le codage manuel (Annexes 2 et 3) que nous avons effectué sur l'ensemble des trente entretiens nous permet de relater ici les verbatim relatifs à ces huit entretiens, définis par notre codage, comme les plus représentatifs des liens entre tensions et proximités exprimés par l'ensemble des personnels interrogés.

2 grands thèmes :

- Les tensions dans l'établissement,

- La place des proximités et ses éventuels effets ces tensions.

Tableau 1 : caractéristiques des personnes interrogées dont les propos ont été repris pour illustration des résultats (source : auteurs)

\begin{tabular}{|l|l|l|l|}
\hline \multicolumn{1}{|c|}{ Code } & \multicolumn{1}{|c|}{ Type de personnel } & Périmètre d'encadrement & $\begin{array}{c}\text { Durée de } \\
\text { l'entretien }\end{array}$ \\
\hline E1 Mme A & $\begin{array}{l}\text { Directrice des soins, de formation } \\
\text { infirmière, titulaire d'un Master } \\
\text { management et gestion des } \\
\text { établissements de santé et d'un } \\
\text { diplôme d'université d'éthique }\end{array}$ & $\begin{array}{l}\text { Encadrement de 400 } \\
\text { personnes }\end{array}$ & $1 \mathrm{~h} 05$ \\
\hline E2 Mme B & Médecin oncologue (DIM) & $\begin{array}{l}\text { Très impliquée dans des } \\
\text { missions transversales dont } \\
\text { la démarche qualité }\end{array}$ & $1 \mathrm{~h} 20$ \\
\hline E3 M. C & Chirurgien, chef de service & & $48 \mathrm{mn}$ \\
\hline E4 Mme D. & $\begin{array}{l}\text { Directrice financière en charge des } \\
\text { finances, du contrôle de gestion, } \\
\text { des litiges, des dons et legs }\end{array}$ & $\begin{array}{l}\text { Pilotage d'un service de 9 } \\
\text { personnes }\end{array}$ & $40 \mathrm{mn}$ \\
\hline E5 M. E. & Infirmier réanimation et expert & & $30 \mathrm{mn}$ \\
\hline
\end{tabular}




\begin{tabular}{|l|l|l|l|}
\hline E6 M. F. & $\begin{array}{l}\text { Cadre de département, Infirmier } \\
\text { anesthésiste, MBA en santé }\end{array}$ & $\begin{array}{l}\text { Encadrement de 100 } \\
\text { personnes (paramédicaux, } \\
\text { soignants médico technique } \\
\text { manipulateurs radio }\end{array}$ & $58 \mathrm{mn}$ \\
\hline E7 M. G. & Cadre administratif logistique & & $42 \mathrm{mn}$ \\
\hline E8 Mme H. & $\begin{array}{l}\text { Assistante de la direction adjointe, } \\
\text { assistante médicale }\end{array}$ & & $36 \mathrm{mn}$ \\
\hline
\end{tabular}

\section{Principaux résultats}

Les propos des personnes interrogées permettent de constater la prégnance des tensions éthiques, organisationnelles, managériales et artefactuelles dans l'univers hospitalier mais aussi de constater, dans une visée managériale, la place des proximités dans l'apparition, l'atténuation ou la disparition de ces tensions.

\subsection{La délicate articulation de l'organisation interne entre les fonctions support et fonctions opérationnelles}

Les tensions managériales apparaissent nettement dans le discours des personnes interrogées. Elle se manifestent plus particulièrement chez certains des professionnels interrogés qui ont fait seulement une grande partie de leur carrière dans l'établissement étudié et ont été confrontés à d'autres contextes professionnels. Pour ceux-ci, les fonctions opérationnelles (médicales et de soins) sont très souvent au service des fonctions support et non l'inverse, ce qui se traduit par une forme d'incompréhension réciproque du rôle de chacun renvoyant à une certaine distance cognitive. Les personnes n'exerçant pas le même métier ne se comprennent pas. Du point de vue d'une partie des interrogés, ces tensions managériales sont amplifiées par la diffusion de processus managériaux centrés sur le « rendre compte » (accountability). Cela est particulièrement prégnant pour les cadres de proximité et les personnels soignants. Cette situation se traduit concrètement par une modification de l'activité pour ces catégories de personnels qui se perçoivent comme assumant une partie du rôle des gestionnaires. La part de l'activité des personnels centrée sur l'acte médical et l'acte de soin s'atténue au profit d'une activité de nature plus administrative (remontées de données d'activité) pour laquelle ces personnels n'ont pas été formés. Ainsi, il apparait que les personnels médicaux et soignants ne s'emparent pas naturellement des fonctions de gestion mais qu'ils les subissent comme des formes d'injonction dans une démarche prescrite. Cette évolution marque selon eux une altération de la proximité sociale avec les autres personnels et les patients du fait de la réduction du temps à leur contact et questionne dans une certaine mesure l'éthique de leur cœur de métier. En effet, les cadres, les personnels médicaux et soignants disent se questionner dans ce contexte sur le sens de leurs actions.

"Ce qui pêche actuellement dans le système des hôpitaux et qui rend les gens un peu fous c'est que le terrain est sans cesse obligé de rendre des comptes sur le plan administratif, sur le plan d'une certification, des rapports à établir et les fonctions ne sont plus des fonctions support mais des fonctions auprès desquelles le terrain doit sans cesse rendre des comptes, on se met alors à dysfonctionner car on perd le sens de l'activité auprès du malade. Ce qui crée l'activité c'est le médecin, toute l'activité de soin auprès du patient. Aujourd'hui, la mise en place de cette technocratie fait que les choses se sont inversées non seulement on doit s'occuper des malades mais aussi rendre des comptes sans cesse et donc on se dit : notre travail consiste en quoi ? et on est en perte de sens. »(E1)

Cependant, dans le CLCC objet de la présente recherche, ces tensions managériales semblent en partie réduites par au moins deux formes de proximité : 
- Une proximité institutionnelle fondée sur des valeurs communes que les représentants des fonctions support décrivent par leur attachement aux valeurs soignantes de leur établissement qui consistent pour eux à être au service des fonctions médicales afin de leur apporter le soutien nécessaire à l'action, même si certains notent une évolution des valeurs en partie liée à une conjoncture difficile («les valeurs n'étaient pas exactement les mêmes il y a 15 ans, la T2A, les coupes budgétaires, la fracture intergénérationnelle transforment l'ambiance de travail et altèrent le climat ») (E7).

- Une proximité cognitive créée à partir de connaissances managériales communes et partagées. Même si on peut imaginer que l'introduction de nouveaux instruments (tels que la tarification à l'activité, il y a quelques années) puisse être source de tensions d'abord artefactuelles et ensuite managériales, dans la durée. Ceux-ci rapprochent plus qu'ils n'éloignent les fonctions financières et la direction générale tenue par un médecin. Avec le temps, ces dispositifs créent des langages communs même si certaines tensions perdurent telles que celles éthiques.

Ainsi l'introduction de nouveaux instruments (indicateurs, tableaux de bord) permet progressivement de transformer les pratiques managériales et en facilite leur acceptation. Le langage de ces mondes différents («Il existe un cloisonnement entre les services étant donné la technicité des services. »(E8)) peut être source d'incompréhensions et de tensions. Cependant, il existe une cohésion forte dont les racines se trouvent dans l'effort pédagogique des différents corps professionnels les uns envers les autres destiné finalement à développer une certaine proximité cognitive.

"L'arrivée de la tarification à l'activité a fait que l'ancien directeur médecin a dî se familiariser avec le contrôle de gestion et est venu vers moi. J'ai toujours eu une relation de proximité, l'argent étant le nerf de la guerre. » (E4)

«Petit à petit il a fallu impliquer les personnels médicaux, et c'est grâce à cela qu'on a pu faire ce que l'on a fait. » (E4)

Le partage de nouveaux outils et la transparence sur leur signification et leur usage est perçue comme un moyen d'amenuiser les tensions managériales et permet l'instauration de la confiance à condition de dépasser le stade du changement perçu comme difficile.

« J'ai accès à la comptabilité analytique du service et cela renforce la confiance tout en augmentant la responsabilité. »(E6).

\subsection{Le rôle ambivalent de la codification des pratiques}

Il apparait clairement que les différences, voire la spécialisation extrême, des pratiques dans un univers complexe peut créer des tensions organisationnelles liées à la coordination des équipes. La mise en place de normes et processus peut représenter une voie pour faciliter la coopération des acteurs internes. Même si leur mise en place et leur déploiement peuvent se traduire en préambule par des tensions artefactuelles, ils contribuent dans la durée à créer un registre de pratiques communes, un «langage commun», qui permet de renforcer la dimension cognitive de la proximité.

"Il est important que les processus de prise en charge soit parfaitement uniformes car cela rassure le patient et est générateur de confiance. Par exemple s'il voit une infirmière désinfecter une plaie une fois avec le bétadine, une fois de biseptine et celle d'après avec un autre produit, le patient peut se demander mais pourquoi elles changent de produits. »(E1)

Comme l'indique plusieurs personnels de direction et soignants, il est important de penser en terme de continuité dans la nature des soins, en appliquant des protocoles similaires qui 
constituent finalement un langage commun. Ces processus constituent aussi une forme de moyens de contrôle plus aisément acceptée, car ces procédures sont rassurantes. La mise en place des démarches qualité est de ce point de vue-là très illustrative. Si lors de leur introduction, ces démarches ont pu être source de tensions artefactuelles et le sont encore, elles rapprochent autour un langage commun (proximité cognitive) mais se diffusent plus ou moins rapidement selon les corps professionnels.

«Avec la qualité, on est parti de zéro mais on a maintenant un niveau de maturité qui fait qu'on est beaucoup moins dans la procédure pour respecter seulement une démarche qualité mais véritablement pour la gestion des risques. »(E2)

«Maintenant tout doit être écrit, tout doit être tracé, et ça c'est un progrès indéniable, un vrai langage commun qui facilite la transmission envers les jeunes. Ces règles en créant des réflexes libèrent de l'espace, du temps et de la disponibilité »(E3)

«Le but est d'intégrer la gestion des risques dans la pratique quotidienne, un volet qui a été donné aux praticiens mais ce n'est pas neutre. Les internes n'y ont pas été formés, ils découvrent cela sur le terrain et le voient encore de manière contraignante». (E2) "La communauté médicale est un peu plus difficile à faire évoluer sur ce plan de la qualité que les soignants. »(E2)

Après avoir été source de tensions artefactuelle et managériale lors de leur introduction, les personnels s'approprient progressivement la norme et la procédure qui finalement les rapprochent.

"C'est le changement qui est difficile quand on vous oblige à faire différemment, ̀̀ changer vos méthodes, ça arrive mais ça met du temps. »(E8)

"J'ai deux ressentis, parfois je ne comprends pas les nouvelles procédures et en même temps il faut que les choses évoluent. Il faut avoir un esprit assez large pour accepter cela, dans notre métier comme pour les médecins c'est important que nos façons de faire se transforment car la médecine et les techniques évoluent. »(E5).

\subsection{Les relations inter-organisationnelles, la proximité comme source de tensions multiples}

Sur un autre plan, la mise en place de dispositifs particuliers s'inscrit dans une approche de l'organisation plus large que l'hôpital lui-même. Les consultations avancées réalisées par les médecins de spécialités dans des hôpitaux parfois très distants contribuent à réduire les tensions éthiques (accessibilité de tous à l'expertise médiale). Comme l'indique les médecins du CLCC interrogés, cette mise en place contribue à atténuer le déficit de ressources médicales dans certaines zones et à réintroduire entre le corps médical et le patient, mais aussi entre les professionnels situés dans des zones géographiques distantes, une forme de proximité à travers ces dispositifs.

"Les médecins du CLCC viennent à la rescousse dans les zones désertifiées pour aider les collègues en sous-effectif. Il y a une organisation de type plateforme qui a prévalu pour la radiothérapie et pour l'oncologie une organisation centrée sur une mission d'appui telles que les consultations avancées. »(E2)

Les Groupements Hospitaliers de Territoire apparaissent comme une solution pilotée par les CHU pour recréer ou renforcer une forme de proximité organisationnelle dans la coordination du suivi des parcours des patients. Cependant, le rôle et le pouvoir des CHU dans le cadre des GHT reste encore flou, et la manière selon laquelle les CHU exerceront cette forme de pouvoir ne sera pas neutre sur les tensions de nature managériales qui pourraient naitre entre les établissements.

Le scénario parfois évoquée du groupement de plusieurs cliniques à l'échelle de la Métropole, 
la possible fusion entre deux CLCC régionaux, ou encore l'hypothèse de rapprochement entre le CLCC et le CHU questionnent directement le périmètre organisationnel et constitue une source de préoccupations pour la plupart des personnels, (toutes catégories confondues) qui peut être à l'origine de tensions éthiques (certains personnels évoquant le risque de créer une distance vis-à-vis du patient). La question de la place du CLCC dans ces configurations organisationnelles projetées interroge directement son pouvoir dans un nouvel ensemble.

«Lorsque le CHU a créé la nouvelle aile de son bâtiment et la passerelle avec le CLCC, cela a ravivé les inquiétudes » (E8)

Ainsi, comme l'illustre ce propos, des éléments même symboliques de proximité géographique ravivent les tensions. Cette nouvelle construction est perçue par des personnels comme un pas vers un rapprochement organisationnel voire les signaux faibles d'une fusion et ils craignent si ce scenario était envisageable une dilution des spécificités de fonctionnement des CLCC.

\subsection{Une proximité géographique pour amenuiser les tensions mais de manière très localisée}

Cependant, il ressort aussi à l'opposé que la proximité des établissements CLCC et CHU permet d'amenuiser les tensions organisationnelles qui pourraient trouver leur origine dans une concurrence sur un territoire donné. En concertation, les établissements concentrent leurs activités sur certaines pathologies, ce qui se traduit par une forme de partage d'activités. Les deux types d'établissement partagent une vision commune de leur mission. Le CLCC bien qu'étant un établissement privé a une mission de service public, de recherche et de formation similaires à celles des $\mathrm{CHU}$ ce qui rapproche les deux établissements. De même les établissements partagent un certain nombre de moyens (plateaux techniques, spécialistes) grâce à la proximité géographique qui agit sur la proximité sociale (facilitation des échanges et interactions entre les personnels des deux établissements).

«La coopération entre le CLCC et le CHU est ici assez naturelle «...». La coopération avec d'autres établissements plus distants est plus difficile mais cela dépend si la démarche est volontaire ou imposée. »(E2)

Néanmoins, le rapprochement avec des établissements plus lointains ou différents est vécu comme une véritable menace organisationnelle qui se traduirait par une transformation des modes de fonctionnement, des pratiques managériales, ce qui générerait plus particulièrement des tensions managériales et artéfactuelles.

"La fusion avec le centre Léon Bérard à Lyon était source de tensions, leur organisation étant très différente, les patients n'étant pas les mêmes... »(E2)

\subsection{Les CLCC, un modèle de direction appréciée légitimant le changement}

Le modèle organisationnel des Centres de Lutte Contre le Cancer constitue une vraie particularité. La direction générale est assurée par un médecin et non pas par un directeur manager ce qui pour les personnes interrogées garantit une proximité institutionnelle peu commune dans les autres types d'établissements médicaux et de soins dirigés par des gestionnaires et relativement coupés du corps médical.

«La proximité entre la direction et l'encadrement et le terrain c'est quand même la défense d'un modèle de prise en charge et d'un modèle d'organisation. »(E1)

«Il y a d'excellents rapports entre la direction et les praticiens. On dispose de paramètres économiques qui sont fondamentaux et nous permettent d'avancer en termes d'adaptation de nos indicateurs de résultat. »(E3) 
«Le fait d'avoir une direction médicale facilite les relations, on parle le même langage, on parle à un confrère. C'est un médecin avant d'être un directeur ». (E2)

«Le fait que la production, c'est-à-dire le médical puisque le centre est dirigé par un médecin, est à l'origine du projet stratégique est intéressant. » (E4)

«Traditionnellement, les médecins se méfient des dirigeants, mais le fait d'avoir à notre tête un médecin change les choses. »(E6)

La taille du CLCC qualifiée de «à taille humaine contribue à gommer les distances hiérarchiques et facilite les échanges »(E5) entre les différentes catégories de personnels même s'il existe différents types de langages (médical, soignant, gestionnaire). Ainsi, les tensions qu'évoquent les interviews seraient amplifiées par un rapprochement ou une fusion entre deux établissements (entre deux CLCC, ou CLCC et CHU). Une taille plus importante générerait des tensions organisationnelles, managériales et artéfactuelles qui ne pourrait finalement pas être atténuée par une proximité sociale altérée du fait de la taille plus importante des établissements.

\subsection{Tensions organisationnelles, quand la transversalité interroge la proximité}

S'il n'existe pas de tensions organisationnelles fortes (pour le CLCC étudié) dans les liens qu'entretiennent les services support et ceux à l'origine de l'activité médicale, celles-ci sont détectables lorsqu'est évoquée l'évolution de l'organisation vers plus de transversalité. En effet, le secteur de la cancérologie est marqué par une prise en charge particulièrement complexe nécessitant l'intervention d'une multitude de personnels parfois appartenant à une même profession. Ainsi, bien qu'appartenant à un même corps professionnel, par exemple celui des personnels infirmiers, l'introduction de la transversalité se traduit par l'intervention de professionnels extérieurs au service dans lequel œuvrent des personnels, que nous pouvons qualifier de sédentaires, au périmètre (ici au sens d'espace physique) bien défini, institué. Celle-ci produit alors des tensions organisationnelles amplifiées par une distance sociale et organisationnelle antérieure.

«Le plus difficile est d'instaurer la transversalité car ce n'est pas naturel. Ça n'inspire pas la confiance, les infirmières ont leur champ de compétence mais le fait d'avoir des infirmiers qui viennent des départements des soins de support, des diététiciens, cela percute l'organisation. " (E1)

«Il est important que les personnels se connaissent et connaissent le travail de l'autre pour amenuiser les tensions au sein et entre les services médicaux et de soins. »(E5)

Cette situation nous montre que la présence de la proximité cognitive et de celle institutionnelle ne suffisent pas à limiter les tensions organisationnelles. Il peut exister une tension entre ces personnels soignants « sédentaires » et «itinérants » (au sein du centre) bien que ceux-ci appartiennent à une même organisation (le centre de lutte contre le cancer). Ce qui amène à considérer que la proximité organisationnelle est peut-être plus à considérer au niveau du département, voire même du service, que du CLCC ou du GHT.

Sur un autre plan, celui des nouvelles formes d'organisation des parcours patient impulsées par les progrès et les innovations thérapeutiques mais aussi dictées par des impératifs économiques avec le développement de l'ambulatoire, il ressort une altération marquée de la proximité géographique qui dans le cas présent peut être source, par ailleurs, de tensions éthiques.

"Ce qui rend plus délicat la création d'une proximité avec le patient et que celui-ci va et vient entre diverses organisations publiques, privées sur un territoire, il passe moins de temps à l'intérieur de l'hôpital, nous l'avons moins sous la main. »(E1)

Ces évolutions vont de pair pour les interrogés avec un amenuisement de la proximité 
relationnelle. Cette dernière repose depuis des siècles sur une relation dissymétrique admise entre le médecin et le patient et qui ne donnait pas lieu à tensions.

«Avec la chronicisation de la maladie les malades, pour une partie d'entre eux, acquièrent au fil des mois une connaissance et du coup le rapport du patient et $d u$ professionnel a évolué. On n'est plus uniquement dans le professionnel sait pour le patient, le patient a aujourd'hui des choses à nous apprendre, il développe une expertise et il faut apprendre aux professionnels à se positionner. »(E1)

Le relâchement de la proximité géographique avec la diffusion de nouvelles formes de prise en charge génère des tensions de nature organisationnelle et artefactuelle et interroge sur la manière de recréer une proximité qui s'appuie traditionnellement sur la densité d'échanges autour d'un geste très technique. Assez paradoxalement, alors qu'il existe un véritable engouement autour des moyens pour permettre un suivi de qualité du patient à distance, les objets connectés, dispositifs de suivis et la e-santé ne sont pas spontanément évoqués ${ }^{2}$.

«En cancérologie il y a vraiment cette complémentarité sur une densité de soins très importante avec une technicité très forte. »(E2)

"On a beau être expert, avec les patients et à leur contact, on apprend tous les jours. »(E3)

"Le soin, en tant qu'acte technique, est un moyen pour entrer en communication, pour entrer dans la relation avec le patient. »(E1)

Ce relâchement du lien de proximité physique avec le développement des suivis à distance pose aussi la question centrale de la coordination des acteurs d'un parcours au niveau d'un territoire de santé et du pouvoir qui lui est associé. La coordination des parcours place celui qui en est l'orchestre dans une situation de pouvoir dans la mesure ou son action peut impacter le volume d'activité des structures et professionnels intervenants dans le parcours de soin. Ces nouvelles formes de prise en charge suscitent des tensions organisationnelles et artefactuelles dans la mesure où il s'agit de ne plus penser en termes d'activités (nombre d'actes) mais de parcours patient, même si cela n'est pas dit explicitement par les répondants, cette idée est bien présente.

Parallèlement à cela, certains patients acquièrent des connaissances et des compétences du fait de leur autonomisation ce qui peut contribuer à créer une proximité cognitive dans la durée mais exige des personnels de revoir leur positionnement professionnel et d'adopter de nouvelles pratiques et de nouveaux outils de suivi. Ce dernier point ne semble pas être associé à des tensions artefactuelles. Dans ce cas précis, les professionnels sont ouverts à l'adoption de nouveaux instruments de suivi. Il s'agit donc pour les répondants d' "Apprendre à gérer la proximité dans un temps beaucoup plus immédiat et beaucoup plus court. " "Les temps interactions effectifs sont de plus en plus courts ce qui offre moins de plages utilisables pour construire la confiance interpersonnelle. »(E1)

\section{Propos conclusifs et apports de la recherche}

Notre objectif de recherche était d'analyser en quoi les proximités entre les acteurs d'un CLCC Jean Perrin peuvent permettre de caractériser les tensions propres à un établissement de santé. A partir d'une grille de lecture combinant proximités et tensions, nous avons essayé de qualifier le rôle des proximités dans la survenance des tensions de gouvernance en identifiant, quelles formes de proximités déterminent, par leur présence ou leur absence, l'apparition de ces tensions (tableau 2). Nous présentons par quels facteurs de

\footnotetext{
${ }^{2}$ Rappelons que ce point ne figurait pas dans le guide d'entretien mais il aurait pu être évoqué comme un moyen de recréer (ou pas) de la proximité, et être abordé comme source (ou pas) de tensions.
} 
l'environnement interne (outils de gestion, procédures, valeurs, spécialisation) et externe (rapprochement organisationnel, accountability) de l'établissement étudié, chacune des proximités peut produire des effets sur les tensions de gouvernance. A ce stade, nous ne pouvons mettre en évidence que des effets positifs (en noir), réduisant les tensions, ou négatifs (en rouge), les amplifiant. Nous pouvons également identifier une hiérarchie entre des proximités produisant des effets sur l'ensemble des tensions et d'autres n'en impactant seulement que quelques formes.

Il s'agit d'un premier travail de mise en relation des proximités et des tensions qui indique et justifie l'existence de liens étroits entre ces deux éléments. La prochaine étape de ce programme de recherche devra nous amener à analyser, d'une part, la persistance ou le changement, dans le temps, des facteurs de proximité dans les établissements de santé produisant ou réduisant les tensions et, d'autre part, les dynamiques entre les proximités qui peuvent se créer pour annihiler ou amplifier certains effets définis dans cette première étude.

Tableau 2. Les effets des proximités sur les tensions de gouvernance (source : auteurs)

\begin{tabular}{|c|c|c|c|c|}
\hline $\begin{array}{l}\text { Tensions } \\
\text { Proximités }\end{array}$ & managériales & éthiques & organisationnelles & artefactuelles \\
\hline $\begin{array}{c}\text { Proximité } \\
\text { géographique }\end{array}$ & & & $\begin{array}{l}\text { Outils de la e- } \\
\text { santé et suivi des } \\
\text { parcours de soin }\end{array}$ & $\begin{array}{l}\text { Outils de la e-santé } \\
\text { et suivi des } \\
\text { parcours de soin }\end{array}$ \\
\hline $\begin{array}{l}\text { Proximité } \\
\text { cognitive }\end{array}$ & $\begin{array}{c}\text { Spécialisation } \\
\text { des fonctions à } \\
\text { l'hôpital }\end{array}$ & $\begin{array}{c}\text { Spécialisation } \\
\text { des fonctions à } \\
\text { l'hôpital }\end{array}$ & $\begin{array}{l}\text { Spécialisation des } \\
\text { fonctions à } \\
\text { l'hôpital }\end{array}$ & $\begin{array}{c}\text { Procédures de } \\
\text { travail communes } \\
\text { (e.g. qualité) }\end{array}$ \\
\hline $\begin{array}{c}\text { Proximité } \\
\text { organisationnelle }\end{array}$ & $\begin{array}{c}\text { Rapproche } \\
\text {-ment des } \\
\text { établissements }\end{array}$ & $\begin{array}{l}\text { Rapproche } \\
\text {-ment des } \\
\text { établissements }\end{array}$ & $\begin{array}{c}\text { Rapprochement } \\
\text { des établissements }\end{array}$ & \\
\hline $\begin{array}{c}\text { Proximité } \\
\text { institutionnelle }\end{array}$ & $\begin{array}{c}\text { Valeurs } \\
\text { soignantes }\end{array}$ & $\begin{array}{c}\text { Valeurs } \\
\text { Soignantes }\end{array}$ & $\begin{array}{c}\text { Valeurs } \\
\text { spécifiques des } \\
\text { CLCC }\end{array}$ & $\begin{array}{c}\text { Procédures de } \\
\text { travail communes } \\
\text { (e.g. qualité) }\end{array}$ \\
\hline Proximité sociale & $\begin{array}{l}\text { Accountability } \\
\text { (rendre des } \\
\text { comptes) }\end{array}$ & $\begin{array}{l}\text { Accountability } \\
\text { (rendre des } \\
\text { comptes) }\end{array}$ & & \\
\hline
\end{tabular}

Ainsi, plus en détail, l'application du cadre de l'Ecole de la Proximité sur l'analyse des TGP dans le CLCC étudié révèle au moins quatre constatations importantes.

- Les proximités peuvent avoir des effets positifs (en noir) ou négatifs (en rouge) (tableau 2) sur les quatre formes de tensions illustrées dans la littérature. Ceci donne à la proximité un rôle moins vertueux que celui que l'on pourrait lui attribuer au premier abord. Ceci rejoint les résultats obtenus en milieu industriel par l'Ecole de la Proximité qui suggèrent l'existence de phénomènes de lock-in et produits par les proximités ou leurs absences. En raison, d'une trop grande proximité entre les acteurs, le lock-in (Rallet et Torre, 2004) maintient les acteurs d'un établissement dans une relation de dépendance qui est source de tensions. Le lock-out (Cohendet et Mehouachi, 2018) ne permet pas de se rapprocher des autres du fait d'une trop grande distance qu'elle soit géographique, cognitive, institutionnelle, organisationnelle ou sociale. Ainsi, dans notre étude de cas, il s'avère que des instruments pourtant soucieux de rapprocher géographiquement (outils de la e-santé et télémédecine, suivi du parcours patient) ou socialement (rendre des comptes en équipe) les acteurs de la santé provoquent surtout des inquiétudes et des tensions organisationnelles et artefactuelles. 
- Dans l'établissement étudié, il semble que ce sont les proximités institutionnelle, cognitive et organisationnelle qui impactent majoritairement les tensions de gouvernance (tableau 2). $\mathrm{Au}$ contraire, les proximités géographique et sociale, qui sont pourtant les formes de rapprochement qui viennent immédiatement à l'esprit quand on parle de proximité produisent des effets réduits sur l'existence ou non de tensions. L'éloignement social et géographique des personnels constitue un changement récent constaté en milieu hospitalier par d'autres travaux (Mériade et al, op.cit.; Pougnet et al., 2016) et qui s'expliquent par l'apparition dans ces établissements d'une plus grande spécialisation des fonctions, technicité des pratiques (e-santé et télémédecine) et reconfiguration des modèles organisationnel et de management (GHT, T2A, parcours patient). Il apparait que la survenance de ces changements de manière simultanée et non coordonnée puisse être une source de tensions que la tutelle et les gestionnaires des établissements doivent prendre en compte dans la gestion du rythme et de l'intensité des réformes.

- Notre étude fait apparaitre de manière très distincte que, dans le cas étudié, la proximité institutionnelle tient un rôle essentiel dans la réduction des tensions de gouvernance. Les valeurs partagées par les personnels (valeurs soignantes, culture d'entreprise, normes professionnelles identiques) semblent être des outils déterminants dans la gestion de ces tensions car elles permettent aux acteurs de se rapprocher autour de principes d'actions communs et partagés (tableau 2). En suggérant qu'aux yeux des personnels de ces établissements, les valeurs soignantes et humaines devraient précéder et déterminer l'atteinte des valeurs économiques, ce résultat complètent des travaux antérieurs (Minvielle et al., 2008; Sicotte et al. , 1998) en précisant comment il est possible, à l'hôpital, d'obtenir un équilibre entre les valeurs humaines et celles économiques que ces derniers travaux appellent de leurs vœux.

- Enfin, les projets de rapprochement d'établissements ou de création de territoires de santé (tels qui sont préconisées par le Plan Santé 2022 en France) présente un caractère ambivalent en termes de tensions de gouvernance (tableau 2). Même s'ils présentent des intérêts importants pour le patient et sa prise en charge, ils sont perçus par les personnels comme des formes organisationnelles imposées et génératrices de tensions notamment sur le plan managérial et éthique. Nos résultats montrent que ces nouvelles organisations des soins devraient avant tout être perçues par les acteurs hospitaliers comme des instruments destinés à améliorer la coopération entre les établissements et réduisant les tensions organisationnelles. En milieu hospitalier, comme cela avait été vérifié en milieu industriel (Boschma, 2005), la proximité organisationnelle présente des avantages dès lors qu'elle a des visées principalement organisationnelles (faire travailler des gens ensemble) et non managériales (par exemple, mutualiser des moyens ou établir une hiérarchie entre établissements).

Notre étude a été réalisée dans un contexte organisationnel spécifique (CLCC) mais représentatif de la réalisation des missions de service public hospitalier en France. Les CLCC accomplissent ces missions selon des logiques de l'Etat providence à partir d'un statut à but totalement non lucratif. Ceci rend leur intervention très proche des situations dans lesquelles le modèle des TGP a été construit. Cependant, ce contexte présente des particularités en termes de proximité dont il sera important de vérifier la récurrence dans d'autres contextes hospitaliers. Par ailleurs, dans cet article, nous avons seulement retenu les réponses les plus significatives et représentatives obtenus de nos interviews et données secondaires. Comme précisé plus haut, nous poursuivons ce travail à travers l'approfondissement d'un programme de recherche plus vaste, mené dans cet établissement, et qui vise à progressivement identifier des moyens d'action pour gérer ces tensions et faciliter la construction de coopérations entre acteurs. Nous avons relaté ici la première étape de ce programme destiné à valider l'intérêt de l'analyse des proximités dans la caractérisation des tensions dans les établissements de santé. 
Ce travail soutient notre présomption initiale, et confirmée par notre étude, selon laquelle l'approche par les proximités peut trouver une place importante dans l'analyse de l'organisation et de la gestion des établissements hospitaliers et ainsi mieux appréhender comment ces mondes en présence peuvent parvenir à évoluer dans un cadre plus serein aux travers de nouvelles dynamiques de proximité (restant à définir plus précisément) et de coopérations. Cela, plus particulièrement aujourd'hui, où de nombreux changements interviennent parallèlement dans ces structures (introduction de la e-santé, rapprochement des établissements, développement de l'ambulatoire, logique du parcours de soin) et modifient, de manière très significative, les formes d'interactions humaines.

\section{Bibliographie indicative}

Angelé-Halgand N., Garrot T., 2015, «Discipliner par le chiffre : l'hôpital financiarisé au risque de la réification ? », Entreprise et Histoire, $\mathrm{N}^{\circ} 79$, Juin, p. 41-58.

Balland P.A., Boschma R., Frenken K., 2015, « Proximity and Innovation: From Statics To Dynamics », Regional Studies, 49, 6, p. 907-920.

Bartoli, A., Mazouz, B., Kéramidas, O., Larat, F., 2011, «Éthique et performance en management public », Revue française d'administration publique, $\mathrm{N}^{\circ} 140$.

Bartoli, A.; Brenkert, G.; Hermel, P., 2012b, «Les défis éthiques de la recherche académique: le cas de la France et des Etats-Unis », Revue française d'administration publique, Vol.140, Éthique et performance en management public, p. 659-675

Bellet M., Colletis G., Lun, Y., 1993, «Economie De Proximités », Numéro Spécial De La Revue d'Économie Régionale et Urbaine, $\mathrm{n}^{\circ} 3$.

Bérard, É., Flachère, I., Saulpic, O., \& Zarlowski, P., 2015, Les outils financiers dans les hôpitaux: penser leur déploiement au-delà de la dimension technique. Journal de gestion et d'économie médicales, 33(7), p. 409-427.

Berard, F., 2010, Tarification à l'activité et nouvelle gouvernance. Éthique \& Santé, 7(3), p. 141-147.

Boschma R.A., 2005, « Proximity and innovation. A critical assessment », Regional Studies, 39, 1, p. 61-74.

Bouba-Olga O. ; Carrincazeaux C. ; Coris M. (eds), 2008, «La proximité : 15 ans déjà !", Numéro spécial, Revue d'Economie Régionale et Urbaine, 3.

Carassus, D., Favoreu, C., Gardey, D., Marin, P., 2012, «La caractérisation et le management des déviances organisationnelles liées à la mise en œuvre d'une démarche de performance publique : application au contexte public local français », Management International, Vol. 16, $\mathrm{N}^{\circ} 3$.

Cassi L., Plunket A., 2014, « Proximity, network formation and inventive performance: in search of the proximity paradox. » The Annals of Regional Science, 53, 2, p. 395-422.

Cohendet, P., \& Mehouachi, C., 2018, Des territoires géographiques à l'écosystème stratégique. Revue française de gestion, (3), p.155-173.

Dupuy C., Torre, A., 2004, «Confiance et proximité », dans Pecqueur et Zimmermann. (Eds), Economie de Proximités, Hermès, Lavoisier, Paris.

Gilly, J.P. ; Torre, A (éds), 2000. Dynamiques de proximité, L'Harmattan, Paris.

Georgescu I., Naro G., 2012, «Pressions budgétaires à l'hôpital: Une étude qualitative du concept de «rapm» auprès de praticiens hospitaliers.»Comptabilité-Contrôle-Audit, 18, 3, p. 67-95. 
Glouberman, S., \& Mintzberg, H. (2001). Managing the care of health and the cure of disease-Part I: Differentiation. Health care management review, 26(1), 56-69.

Glouberman, S., \& Mintzberg, H. (2001). Managing the care of health and the cure of disease-Part II: Integration. Health care management review, 26(1), 70-84.

Glouberman, S., \& Mintzberg, H. (2002). Gérer les soins de santé et le traitement de la maladie. Gestion, 27(3), 12-22.

Granovetter M., 1985, « Economic Action and Social Structure: The Problem of Embeddedness », American Journal of Sociology, vol.91 n³, novembre, p. 481-510.

Grenier C., 2009, "Le réseau de santé : une lecture constructiviste par la notion de gouvernance conceptive », Journal d'Economie Médicale, vol 27, 1-2, p. 73-89

Grenier, C., \& Ibrahim, R., 2015, La gouvernance auscultée: entre une gouvernance technique qui prescrit et une gouvernance comme espace de renouvellement des relations et des habiletés à innover. Journal de gestion et d'économie médicales, 33(6), p.327-334.

Grossetti M.,2008, Proximities and embedding effects, European Planning Studies, 16, 5, p. 629-642.

Ingham, M., De Viron, F., \& Tihon, A., 2011, «Proximité entre parties prenantes et alignement stratégique ». Revue française de gestion, (4), p. 125-140.

Kirat T., Lung Y., 1995, «Innovations et proximités : le territoire, lieu de déploiement des processus d'apprentissage ", dans N. LAZARIC et J. M. MONNIER (eds.), Coordination économique et apprentissage des firmes, Economica, Paris.

Kirat T., Lung Y., 1999, « Innovation and proximity. Territories as loci of collective learning processes », European Urban and Regional Studies, 6, 1, p. 27-38.

Knoben J., Oerlemans L., 2006, " Proximity and inter-organizational collaboration: a literature review », International Journal of Management Reviews, 8, 2, p. 71-89.

Marival C., Petrella F. et Richez-Battesti N., 2015, «Coopération et gouvernance: Normalisation ou reconquête du fait associatif?», Journal de gestion et d'économie médicales, 33(6), p. 359-373.

Mazouz, B., Tardif, M., 2010, « À propos de la performance : l'Arlésienne de la sphère publique »dans Proulx, Denis (éds), Management des organisations publiques : théorie et applications. Québec : Presses de l'Université du Québec (2e éd. rev. et corr.), p. 31-61.

Mazouz, B. ,2012, «Gestion par résultats », dans L. Coté et J.-F. Savard (eds), Le Dictionnaire encyclopédique de l'administration publique.

Mazouz, B., Garzon C., Picard, P., 2012, «Les déviances dans les organisations publiques en quête de performance: vers une gestion prophylactique des risques de déviance », Management international, Vol.16, N3, p. 92-100.

Mériade, L., 2017, «L'instrumentation des tensions de gouvernance publique (TGP): L'exemple des simplifications des indicateurs dans le système universitaire français », Management International, $\mathrm{Vol} 21, \mathrm{~N}^{\circ} 4, \mathrm{p} 12-27$.

Mériade, L., Nautre, B., Rochette C., Talbot D., 2017, «Les effets de la Proximité sur l'exécution des politiques publiques: L'exemple d'un Centre de Lutte contre le Cancer», Politiques et Management public, 34/1 janvier -juin 2017, p. 143-162

Minvielle, E., Sicotte, C., Champagne, F., Contandriopoulos, A.-P., Jeantet, M., Préaubert,N., Bourdil, A., Richard, C., 2008, Hospital performance: Competing or shared values? Health Policy 87, p. 8-19.

Nilsson M., Mattes J., 2015, « The spatiality of trust: Factors influencing the creation of trust and the role of face-to-face contacts. » European Management Journal, 33, 4, p. 230-244.

Pecqueur B., Zimmermann J.B. (éds), 2004, Economie de Proximités, Hermès, Lavoisier : Paris.

Pierru F., 2012, La santé en fusions. L'accouchement des agences régionales de santé au forceps institutionnel. Dans : La territorialisation des politiques de santé. Bordeaux : Editions 
Hospitalières, p. 77-108

Pougnet, R., Pougnet, L., Loddé, B., \& Dewitte, J. D., 2016, Travailler dans un hôpital est riche de sens et source de mal-être: analyse qualitative auprès de 9 médecins hospitaliers. $\mathrm{La}$ Presse Médicale, 45(7-8), e233-e241.

Rallet, A., \& Torre, A., 2004, Proximité et localisation. Économie rurale, 280(1), p. 25-41.

Rivière, A., Commeiras, N., \& Loubes, A., 2013, Tensions de rôle et stratégies d'ajustement: une étude auprès de cadres de santé à l'hôpital. Journal de gestion et d'économie médicales, 31(2), p.142-162.

Rychen F., Zimmermann J.B. (eds), 2008, « Clusters in the Global Knowledge-based Economy: Knowledge Gatekeepers and Temporary Proximity », Special Issue of Regional Studies, 42, 6, p. 767-776

Sicotte, C., Champagne, F., Contandrioloulos, A.-P.,Barnsley, J., Béland, F., Leggat, S.G., Denis, J.L., Bilodeau, H., Langley, A., Brémon, M., Baker, G.R., 1998, A conceptual framework for the analysis of Health Care Organizations' performance. Health Services Management Research 11: p. 24-48.

Talbot, D., 2008, Les institutions créatrices de proximités. Revue d'économie régionale \& urbaine, (3), p. 289-310.

Torre A., Gilly J.P., 2000, « On the analytical dimension of proximity dynamics ». Regional Studies, 34, 2, p. 169-180.

Torre A., Rallet A., 2005, « Proximity and localization », Regional Studies, 39, 1, p. 47- 60

Valette, A., \& Burellier, F. (2014). "Quand l'habit fait le moine les chefs des pôles hospitaliers: devenir des hydrides «malgré tout»?» In Annales des Mines-Gérer et comprendre (No. 2, pp. 4-13). ESKA.

Walker, M.R., Damanpour, F., Devece, C.A., 2010, «Management Innovation and Organizational Performance: The Mediating Effect of Performance Management », Journal of Public Administration Research and Theory, Vol. 21, N², p. 367-386.

Wilson, J. M., Boyer O'leary, M., Metiu, A., \& Jett, Q. R., 2008, « Perceived proximity in virtual work: Explaining the paradox of far-but-close». Organization Studies, 29, 7, p. 9791002. 